\title{
Emerging applications of Deep Learning and Spiking ANN
}

\author{
Lazaros S. lliadis $^{1} \cdot$ Chrisina Jayne $^{2}$ \\ Published online: 29 October 2020 \\ (C) Springer-Verlag London Ltd., part of Springer Nature 2020
}

This is the Special Issue on "Emerging applications of Deep Learning (DL) and Spiking Artificial Neural Networks" of the Springer Neural Computing and Applications Journal. It includes seventeen high-quality scientific research papers, presenting innovative research, falling in the above areas. The accepted papers were selected from a large pool of submissions after a peer review process, based on their level of novelty and quality.

Deep Learning belongs to the Machine Learning approaches, and it can be successfully applied even for complex modeling cases with vast amount of diverse or unstructured data, especially in the domains of Image Classification and Natural Language processing. DL is employed in several diverse fields of our everyday life, like self-driving cars, fraud news detection, medical applications, even in social media. On the other hand, Spiking Neural Networks are inspired by the actual function of the brain and more specifically by the emission, communication and processing of pulses known as spikes. They have been proven to be used quite efficiently in control systems (e.g., in Robotics) in cybersecurity modeling and more generally in the development of neuromorphic systems.

The first paper is entitled "Affective Analysis of patients in Homecare Video Assisted Telemedicine using Computational Intelligence," and it is authored by Antonis Kallipolitis, Michael Galliakis both from the Department of Computer Science and Biomedical Informatics, University

\footnotetext{
Lazaros S. Iliadis

liliadis@civil.duth.gr

Chrisina Jayne

C.Jayne@tees.ac.uk
}

1 Laboratory of Mathematics and Informatics (ISCE), Department of Civil Engineering, School of Engineering, Democritus University of Thrace, University Campus, Kimmeria, 68200 Xanthi, Greece

2 Dean of the School of Computing \& Digital Technologies, Centre for Digital Innovation, Senior Management, SCEDT School Executive Team, Teesside University, Campus Heart, Southfield Rd, Middlesbrough TS1 3BX, UK of Thessaly Greece, Andreas Menychtas, Department of Digital Systems, University of Piraeus Greece and Ilias Maglogiannis from the Department of Digital Systems, University of Piraeus Greece.

This paper presents the design, the implementation and the application results of an emotion analysis module integrated in an existing telemedicine platform. The affective/emotional status of patients is strongly connected to the healing process and their health. Therefore, being aware of the psychological peaks and troughs of a patient provides the advantage of timely intervention by specialists or closely related kinsfolk. Two different methodologies are utilized and discussed. The first scheme exploits the fast and consistent properties of the Speeded-Up Robust Features algorithm in order to identify the existence of seven different sentiments in human faces. The second is based on the employment of Convolutional Neural Networks. The whole functionality is provided as a web service for the healthcare platform during regular video teleconference sessions between authorized medical personnel and patients.

The second paper is authored by Zhu Hongpeng, from the Information Engineering Department of Shaoyang University, Hunan, China. The title is "Online MetaLearning Firewall to Prevent Phishing Attacks."

Phishing is the most well-known act of deceiving internet users, in which the "perpetrator" plays a credible entity, by exploiting the ignorance of the user-object, in order to illegally obtain personal sensitive data. This paper introduces the Online Meta-Learning Firewall which is used to prevent phishing attacks. It is an active, highly innovative and fully automated safety tool that employs a long short-term memory meta-learner. It is time effective, as it converges with a fairly small number of steps. The introduced approach is an extension of the hybrid $k$ Nearest Neighbor with Self-Adjusting Memory algorithm.

Ioannis E. Livieris from the Department of Mathematics, University of Patras Greece, Stavros Stavroyiannis, Department of Accounting and Finance University of the Peloponnese, Greece, Emmanuel Pintelas and Panagiotis Pintelas both from the Department of Mathematics, 
University of Patras Greece, are the authors of the third paper. It is entitled "A novel validation framework to enhance deep learning models in time-series forecasting."

This paper introduces a modeling approach on timeseries analysis and forecasting, which is generally considered as one of the most challenging problems of data mining. More specifically, this research effort introduces a novel Deep Learning framework for enhancing the accuracy, efficiency and reliability of time-series models. The major novelty of the proposed model is that it ensures the suitability of a time-series to fit to a Deep Learning model. This is achieved by performing a series of transformations in order to satisfy the stationarity property. The enforcement of stationarity is performed by the application of the Augmented Dickey-Fuller test and by the execution of transformations which are based on first differences or returns, without the loss of any embedded information. The reliability of the obtained predictions is guaranteed by rejecting the hypothesis of autocorrelation in the model's errors. This is demonstrated by autocorrelation function plots and by the execution of the Ljung-Box Q-test. The proposed approach was tested on three real-world application domains (financial market, energy sector, cryptocurrency area) with very promising results.

An interesting research entitled "A Semi-Self-Taught Network Intrusion Detection System" is authored by Feng Zhao, School of Business Administration, Wonkwang University, Korea, Hao Zhang from the Feng Zhao School of Business Administration, Wonkwang University, Korea, and from the Law and Politics Institute, Hebei GEO University China, Jia Peng, from the School of Business Administration, Wonkwang University, Korea, and School of Economics and Management, Xinyu University, China, Xiaohong Zhuang School of Business Administration, Wonkwang University, Korea, and School of Economics and Management, Shangrao Normal University, China, and Sang-Gyun $\mathrm{Na}$, School of Business Administration, Wonkwang University, Korea.

The ever-increasing threat and complexity of modern cyber-attacks require search for integrated and flexible intelligent defense mechanisms. Such approaches can provide optimal countermeasures, reliable credentials extraction and self-adjusting potential. Given the widespread scale of modern networks and the complexity of cyber-attacks, the problem of self-adaptation goes far beyond the capabilities of network Intrusion Detection Systems (IDS). The main weakness of IDS is the fact that they cannot adapt to new network conditions ("zero-day" attacks). This research tries to overcome the above limitation, by introducing a hybrid Semi-Supervised Discriminant Autoencoder (AUE) which combines denoising AUEs with a heuristic method of class separation. In essence, the proposed algorithm learns to remodel the displaced specimens instead of the original ones in the super-sphere defined by their closest neighbors. The purpose is to understand the nature of an attack, based on generalized transformed features derived directly from unknown web environments and data.

The fifth paper is on "An Adversarial Semi-Supervised Approach for Action Recognition from Pose Information." It is authored by George Pikramenos and Eirini Mathe both from the Institute of Informatics and Telecommunications, National Center for Scientific Research "Demokritos," Athens, Greece, Eleanna Vali School of Electrical and Computer Engineering, National Technical University of Athens, Greece, Ioannis Vernikos Institute of Informatics and Telecommunications, National Center for Scientific Research "Demokritos," Athens, Greece, Antonios Papadakis Department of Informatics, University of Athens, Greece, Evaggelos Spyrou Institute of Informatics and Telecommunications, National Center for Scientific Research "Demokritos," Athens, Greece, and Phivos Mylonas Department of Informatics, Ionian University, Corfu Greece.

The collection of video data for action recognition is very susceptible to measurement bias; the equipment used, camera angle and environmental conditions are all factors that majorly affect the distribution of the collected dataset. Inevitably, training a classifier that can successfully generalize to new data becomes a very hard problem, since it is impossible to gather general enough training sets. Recent approaches in the literature attempt to solve this problem by augmenting a given training set, with synthetic data, so as to better represent the global distribution of the covariates. However, these approaches are limited because they essentially involve hand-crafted data synthesizers, which are typically hard to implement and problem specific. This research paper introduces a novel approach to tackling the above issues. It relies on the hybrid combination of pose extraction and domain adaptation as a means to improve the generalization capabilities of classifiers. It has been shown that adapted skeletal representations can be retrieved automatically in a semi-supervised setting which generalizes classifiers to new forms of measurement bias. The proposed model has been empirically validated successfully across different camera angles.

Xie Bing, from the Department of Information Engineering, Shaoyang University, Hunan, China, is the author of the sixth paper. The title is "Critical Infrastructure Protection Based on Memory-Augmented Meta-Learning Framework."

Critical infrastructures are related to systems which are essential for sustaining the important functions of a society. Their potential failures can cause serious problems not only to the population and economy but to national security as well. Traditional security systems, even those that employ 
intelligent algorithms, fail to prevent advanced zero-day attacks as they require constant training. This is a novel and very interesting research that proposes a novel MetaLearning architecture. It considers the Neural Turing Machines as the approach upon which the model is founded. The introduced model allows for the memorization of useful data from past processes, by integrating external storage memory. Moreover, it facilitates the rapid integration of new information without the need for retraining. In particular, the proposed novel architecture is called Memory-Augmented Neural Network. Its core comprises a sophisticated, very fast and highly efficient Extreme Learning Machine. It is assisted by a series of original modifications, related to fine tuning of training, to memory retrieval mechanisms, to addressing techniques, and to ways of attention-weights allocation to memory vectors. The efficiency of the proposed system has been successfully tested using an extremely complex scenario for the protection of critical infrastructures. According to the testing scenario, memory could quickly encode and record information about new types of attacks, while any stored representation from previous experience was easily and consistently accessible, to maximize the detection efficiency.

An interesting research entitled "Building an Efficient OCR System for Historical Documents with Little Training Data" is described in the seventh paper. The authors are Jiří Martínek from the Department of Computer Science and Engineering, Faculty of Applied Sciences, University of West Bohemia, Plzeň, Czech Republic, Ladislav Lenc, from the New Technologies for the Information Society, Faculty of Applied Sciences, University of West Bohemia, Plzeň, Czech Republic and Pavel Král Department of Computer Science and Engineering, Faculty of Applied Sciences, University of West Bohemia, Plzeň, Czech Republic.

As the number of digitized historical documents has increased rapidly during the last few decades, it is necessary to provide efficient methods of information retrieval and knowledge extraction to make the data accessible. Such methods are dependent on optical character recognition (OCR) which converts the document images into textual representations. OCR methods are often not adapted to the historical domain; moreover, they usually need a significant amount of annotated documents. To overcome such limitations, this research paper introduces a set of methods that allow the use of OCR on historical document images using only a small amount of real, manually annotated training data. The presented complete system includes two main tasks: page layout analysis including text block-line segmentation and OCR. This research paper introduces segmentation methods which are based on the employment of Convolutional Neural Networks and OCR that utilizes Recurrent Neural Networks. In the testing process, OCR was performed on a novel real dataset from Porta fontium portal. This corpus is freely available for research. It has been proven that both tasks are feasible with only a few annotated real data samples. The obtained scores are comparable or even better than the ones of several state-of-the-art systems.

The eighth paper is entitled "A deep Q-learning portfolio management framework for the cryptocurrency market," and it is authored by Giorgio Lucarelli from the Department of Economics, Management and Statistics, University of Milan-Bicocca, Italy, and Matteo Borrotti from the Department of Economics, Management and Statistics, University of Milan-Bicocca, Italy.

Deep Reinforcement learning is gaining popularity in many diverse areas. An interesting sector is related to the definition of dynamic decision-making systems. A possible example is dynamic Portfolio Optimization, where an agent has to continuously reallocate an amount of fund into a number of different financial assets. The final goal is maximization of return and minimization of risk. This research proposes a novel deep Q-Learning portfolio management framework. The framework is composed by a set of local agents that learn assets behaviors and a global agent that describes the global reward function. The framework is tested on a crypto portfolio composed by four cryptocurrencies. The obtained results have shown that the Deep Reinforcement portfolio management framework is the optimal approach for dynamic portfolio optimization.

Spiros Georgakopoulos, Sotiris Tasoulis, Georgios Mallis, Aristidis Vrahatis, Vassilis. Plagianakos from the Department of Computer Science and Biomedical Informatics, University of Thessaly, Volos, Greece and Ilias Maglogiannis from the Department of Digital Systems, University of Piraeus, Greece, are the authors of the ninth paper "Change Detection and Convolution Neural Networks for Fall Recognition."

Accurate fall detection is a crucial research challenge since the time delay from fall to first aid is a key factor that determines the consequences. Wearable sensors allow a reliable way for motion tracking, allowing immediate detection of high-risk falls via a Machine Learning framework. Accelerometer devices are widely used for the assessment of fall risk. Although there exist a plethora of studies under this perspective, several challenges still remain, such as dealing simultaneously with extremely demanding data management, power consumption and prediction accuracy. This research paper introduces a hybrid approach based on the cooperation of Deep Learning for signal classification along with a lightweight control chart method for change detection. The basic assumption is that it is possible to control computational resources by selectively allowing the operation of a relatively 
heavyweight, but very efficient classifier, when it is truly required. The proposed methodology was applied to real experimental data providing reliable results that justify the original hypothesis.

The 10th paper "Fake Consumer Review Detection using Deep Neural Networks Integrating Word Embeddings and Emotion Mining" is authored by Petr Hajek, Institute of System Engineering and Informatics, Faculty of Economics and Administration, University of Pardubice, Czech Republic, Aliaksandr Barushka Institute of System Engineering and Informatics, Faculty of Economics and Administration, University of Pardubice, Czech Republic and Michal Munk, Department of Computer Science, Constantine the Philosopher University in Nitra, Slovakia.

Fake consumer review detection has attracted much interest recently, due to the increasing number of Internet purchases. Existing approaches that detect fake consumer reviews mainly use the review content and product-reviewer information. As it has been shown in recent studies, the semantic meaning of reviews might be particularly important for text classification. In addition, the emotions hidden in the reviews may represent another potential indicator of fake content. This research paper introduces two Neural Network models that integrate traditional bagof-words as well as the word context and consumer emotions in an effort to enhance the fake review detection performance. Specifically, the models learn documentlevel representation by using three sets of features, namely: n-grams, word embeddings and lexicon-based emotion indicators. Such a high-dimensional feature representation is used to classify fake reviews into four domains. The proposed approach has been proven to be very efficient and more accurate compared to several state-of-the-art methods.

The 11th paper is entitled "On time series representations for multi-label NILM," and it is authored by Christoforos Nalmpantis and DimitrisVrakas, both from the School of Informatics, Aristotle University of Thessaloniki, Greece.

Given only the main power consumption of a household, a non-intrusive load monitoring (NILM) system identifies which appliances are operating. With the rise of Internet of Things, running energy disaggregation models on the edge is more and more essential for privacy concerns and economic reasons. However, current NILM solutions use data hungry Deep Learning models that can recognize only one device and are not capable of running on advice with limited resources. This research paper investigates in depth multi-label NILM systems, and it suggests a novel framework which enables a cost-effective solution. It has been shown that privacy can be preserved after deploying it on an embedded device. The introduced system leverages dimensionality reduction, using Signal2vec. It has been tested on two popular public datasets, and it has proven its ability to outperform other state-of-the-art multi-label NILM methods.

Serafin Alonso, Antonio Moran, Daniel Perez, Miguel Prada, Manuel Dominguez all from the University of Leon Spain (Grupo de Investigacion en Supervision, Control y Automatizacion de Procesos Industriales) and Ignacio Diaz from the Electrical Engineering Department of the University of Oviedo Spain are the authors of the paper entitled "Estimating cooling production and monitoring efficiency in chillers using a soft sensor."

Intensive use of heating, ventilation and air conditioning systems in buildings entails monitoring their efficiency. Cooling systems are key facilities in large buildings and can account up to $44 \%$ of the energy consumption. Monitoring chillers' efficiency is an important task. Sensors that can be used to measure the cooling production are rarely installed due to their cost. This research paper introduces a methodology to build a soft sensor that provides an estimation of cooling production and enables monitoring the chiller's efficiency. The proposed soft sensor can consider independent variables (internal states of the chiller and electric power), and it can take advantage of current or past observations. Six models (including linear approaches and Deep Learning) have been developed for the implementation of the aforementioned soft sensor. Testing has been performed on datasets obtained by two different chillers (large water-cooled and smaller air-cooled chillers) installed at the Hospital of Leon, Spain.

The authors of the 13th paper are: Rolandos Alexandros Potamias, from the Department of Computing, Imperial College London UK, Georgios Siolas, School of Electrical and Computer Engineering, National Technical University of Athens, Greece, and Andreas-Georgios Stafylopatis, School of Electrical and Computer Engineering, National Technical University of Athens, Greece. The title of the paper is "A Transformer-based approach to Irony and Sarcasm detection."

Figurative Language (FL) seems ubiquitous in all social media discussion forums and chats, posing extra challenges to sentiment analysis endeavors. Identification of FL schemas in short texts remains largely an unresolved issue in the broader field of Natural Language Processing. This is mainly due to their contradictory and metaphorical meaning content. The main FL expression forms are sarcasm, irony and metaphor. This research paper introduces advanced Deep Learning methodologies to tackle the problem of identifying the aforementioned FL forms. The authors are proposing a Neural Network which is based on a recently proposed pre-trained transformer-based Network architecture, which is further enhanced with the employment and devise of a Recurrent Convolutional Neural Network. With this set-up, data preprocessing is kept in 
minimum. The performance of the devised hybrid architecture is tested on four benchmark datasets, and contrasted with other relevant state-of-the-art methodologies and systems. Results demonstrate that the proposed methodology achieves state-of-the-art performance under all benchmark datasets, outperforming, even by a large margin, all other methodologies.

George Kyriakides and Konstantinos Margaritis, both from the Department of Applied Informatics, University of Macedonia, Thessaloniki, Greece, have authored the $14^{\text {th }}$ paper. Its tile is "The effect of reduced training in neural architecture search."

As neural architecture search becomes an increasingly studied field, it has become apparent that it demands a great number of computational resources. These are usually devoted to computations, utilized to train and evaluate inter-mediate solutions during the search phase. Although most researchers focus on developing more efficient search methods, the main computational cost in terms of execution time percentage concerns the evaluation of candidate architectures. As such, many works utilize a smaller number of training epochs during search phase evaluations. This research paper studies the effect of reduced training in neural architecture search. It focuses on the retention of relative rankings between architectures when they are trained with different optimizers and for various epochs. The research has discovered relatively high rank-correlations between various fully and partially trained, arbitrarily connected architectures (Kendall's tau-b > 0.7). These are generated by mutating a simple Convolutional architecture for the CIFAR-10 image recognition dataset. Similar behaviors have been observed in networks sampled from the NAS Bench neural architecture dataset, consisting of a fixed outer skeleton and variable cell module composition. This paper also demonstrates the ability of Genetic algorithms to find optimal solutions in noisy environments, by simulating the previous findings with perturbed n-dimensional Rastrigin functions.

The 15th paper is entitled "Privacy Preserving Distributed Training of Neural Networks," and it is authored by Spyridon Nikolaidis and Ioannis Refanidis, University of Macedonia, Thessaloniki, Greece. This research paper introduces the LEARNAE. It is a system aiming to achieve a fully distributed way of Neural Network training. It follows a "Vires in Numeris" approach, combining the resources of commodity personal computers. It has a full peer-to-peer model of operation; all participating nodes share the exact same privileges and obligations. Another significant feature of LEARNAE is its high degree of fault tolerance. All training data and metadata are propagated through the network using resilient gossip protocols. This robust approach is essential in environments with unreliable connections and frequently changing set of nodes. It is based on a versatile working scheme and supports different roles, depending on processing power and training data availability of each peer. In this way, it allows an expanded application scope, ranging from powerful workstations to online sensors. To maintain a decentralized architecture, all underlying tech should be fully distributed too. LEARNAE's coordinating algorithm is platform-agnostic, but for the purpose of this research the two following novel protocols have been used: IPFS which is a decentralized file system as a means to distribute data in a permissionless environment and IOTA which is a decentralized network, targeting the world of low energy "Internet of Things" devices. This research is an extension of a previous one of the same authors that attempted to search the feasibility of using Distributed Ledger Technology to collaboratively train a Neural Network. In this paper, LEARNAE has been employed towards a fully deployed computer network. This paper focuses on the use of cases that require data privacy.

The 16th paper is entitled "A CNN-LSTM model for gold price time series forecasting," and it is authored by Ioannis Livieris Department of Mathematics, University of Patras, Greece, Emmanuel Pintelas Department of Electrical and Computer Engineering, University of Patras, Greece, and Panagiotis Pintelas Department of Mathematics, University of Patras, Greece. Its title is: "A CNNLSTM model for gold price time series forecasting."

Gold price volatilities have a significant impact on many global financial activities. The development of a reliable prediction model could offer insights into gold price fluctuations, behavior and dynamics. Ultimately it could provide the opportunity of gaining significant profits. This research paper proposes a novel Deep Learning forecasting model for the reliable prediction of gold price and fluctuations. The proposed model exploits the ability of Convolutional layers for extracting useful knowledge and learning the internal representation of time-series data, as well as the effectiveness of long short-term memory (LSTM) layers for identifying short-term and long-term dependencies. A series of testing experiments have been conducted in order to evaluate the proposed model against state-of-the-art Deep Learning and Machine Learning approaches. The preliminary experimental analysis illustrated that the utilization of LSTM layers along with additional Convolutional ones could provide a significant boost in increasing the forecasting performance.

The final 17th paper is authored by Konstantinos Demertzis and Lazaros Iliadis both from the School of Engineering, Democritus University of Thrace, Xanthi, Greece, Nikos Tziritas from the Research Center for Cloud Computing, Shenzhen Institutes of Advanced Technology, Chinese Academy of Sciences, Shenzhen, China, and Panagiotis Kikiras, from the Department of Computer 
Science, School of Science, University of Thessaly, Lamia, Greece. The title of the paper is: "Anomaly detection via blockchained deep learning smart contracts in industry 4.0."

The complexity of threats in the ever-changing environment of modern industry is constantly increasing. At the same time, traditional security systems fail to detect serious threats of increasing depth and duration. This paper introduces alternative, intelligent solutions to detect anomalies in the operating parameters of the infrastructures concerned, while ensuring the anonymity and confidentiality of industrial information. Blockchain is an encrypted, distributed archiving system designed to allow for the creation of real-time $\log$ files that are unequivocally linked. This ensures the security and transparency of transactions. This research presents, for the first time in the literature, an innovative Blockchain Security Architecture that aims to ensure network communication between traded Industrial Internet of Things devices, following the Industry 4.0 standard and based on Deep Learning Smart Contracts. The proposed smart contracts are implementing (via computer programming) a bilateral traffic control agreement to detect anomalies based on a trained Deep Autoencoder Neural Network. This highly novel architecture fuses artificial intelligence in the Blockchain, and it enables the creation of a secure distributed platform that can control and complete associated transactions in critical infrastructure networks, without the intervention of a single central authority.

Publisher's Note Springer Nature remains neutral with regard to jurisdictional claims in published maps and institutional affiliations. 